\title{
Retraction Note to: Semaphorin 4D cooperates with VEGF to promote angiogenesis and tumor progression
}

\author{
Hua Zhou ${ }^{1}$ Nada O. Binmadi ${ }^{1,2} \cdot$ Ying-Hua Yang ${ }^{1} \cdot$ Patrizia Proia ${ }^{1,3} \cdot J^{\prime}$ Jh R. Basile ${ }^{1,4}$
}

Published online: 10 March 2020

(c) Springer Nature B.V. 2020

\section{Retraction to: Angiogenesis (2012) 15:391-407 https://doi.org/10.1007/s10456-012-9268-y}

The Editors-in-Chief have retracted this article [1] following an investigation by the University of Maryland. The institution found that in Fig. 1B and 1D, the cell lines are different and all published histograms show SEMA4D mRNA level whereas Excel data have two histograms showing SEMA4D expression and two histograms showing VEGF expression. In Fig. 2B, the metadata for one image shows different treatment conditions than those reported in the article. The published image labelled "VEGF + VEGFR-2 shRNA" has a metadata label of S4d-plexinB1 shRNA2. In Fig. 2E, statistical significance was shown in the published figure for four comparisons, but upon recalculation, one comparison noted as significant was not. In Fig. 6A, the lower left image is labelled "VEGF shRNA" in the published figure, but the metadata label is "S4DshRNA-HN121-20X". In Fig. 6C, specifically, within columns $2-4$, for each antibody used for immunocytochemistry, the three images have been swapped so that the original images do not match the shRNA labels in the figure (the labels for the two antibodies were correct). In Fig. 7D, the first published image is labelled as "IgG" in the paper, but the metadata show a label of "Restore $(\mathrm{V}+\mathrm{S})$.

The original article can be found online at https://doi.org/10.1007/ s10456-012-9268-y.

John R. Basile

jbasile@umaryland.edu

1 Department of Oncology and Diagnostic Sciences, University of Maryland Dental School, 650 West Baltimore Street, 7-North, Baltimore, MD 21201, USA

2 Department of Oral Basic and Clinical Sciences, King Abdulaziz University, Jeddah 21589, Saudi Arabia

3 Department of Sports Science (DISMOT), University of Palermo, Via M. Toselli, 87/B, 90143 Palermo, Italy

4 Greenebaum Cancer Center, 22 S. Greene Street, Baltimore, MD 21201, USA tif". The third published image has a label of "anti-VEGF IgG", and the metadata show a label of "con sh.tif". Due to these errors, the Editors-in-Chief have found that the results are no longer reliable.

Nada O. Binmadi, Patrizia Proia and John R. Basile agree to this retraction.

Hua Zhou and Ying-Hua Yang have not responded to any correspondence from the publisher about this retraction.

\section{Reference}

1. Zhou H, Binmadi NO, Yang YH, Proia P, Basile JR (2012) Semaphorin 4D cooperates with VEGF to promote angiogenesis and tumor progression. Angiogenesis 15:391-407. https://doi. org/10.1007/s10456-012-9268-y

Publisher's Note Springer Nature remains neutral with regard to jurisdictional claims in published maps and institutional affiliations. 